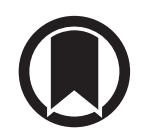

CrossMark

\title{
Pulmonary vascular endothelium: the orchestra conductor in respiratory diseases
}

\author{
Highlights from basic research to therapy
}

Alice Huertas $\mathbb{1}^{1,15}$, Christophe Guignabert $\mathbb{1}^{1,15}$, Joan A. Barberà2,3, Peter Bärtsch ${ }^{4}$, Jahar Bhattacharya ${ }^{5}$, Sunita Bhattacharya ${ }^{5}$, Maria R. Bonsignore ${ }^{6}$, Laurence Dewachter ${ }^{7}$, Anh Tuan Dinh-Xuan ${ }^{8}$, Peter Dorfmüller ${ }^{1}$, Mark T. Gladwin', Marc Humbert (10), Tom Kotsimbos ${ }^{10}$, Theodoros Vassilakopoulos ${ }^{11}$, Olivier Sanchez ${ }^{12}$, Laurent Savale ${ }^{1}$, Ugo Testa $^{13}$ and Martin R. Wilkins ${ }^{14}$

\begin{abstract}
Affiliations: 'INSERM UMR_S 999, Hôpital Marie Lannelongue, Université Paris-Sud/Université Paris-Saclay, AP-HP, Service de Pneumologie, Centre de Référence de l'Hypertension Pulmonaire Sévère, DHU Thorax Innovation, Hôpital Bicêtre, Le Plessis-Robinson and Le Kremlin-Bicêtre, France. ${ }^{2}$ Dept of Pulmonary Medicine, Hospital Clínic, Institut d'Investigacions Biomèdiques August Pi i Sunyer (IDIBAPS), University of Barcelona, Barcelona, Spain. ${ }^{3}$ Centro de Investigación Biomédica en Red Enfermedades Respiratorias (CIBERES), Madrid, Spain. ${ }^{4}$ Dept of Internal Medicine, University Clinic Heidelberg, Heidelberg, Germany. ${ }^{5}$ Lung Biology Lab, Dept of Medicine, Columbia University Medical Center, New York, NY, USA. ${ }^{6}$ Institute of Biomedicine and Molecular Immunology (IBIM), National Research Council (CNR), Biomedical Dept of Internal and Specialistic Medicine (DiBiMIS), University of Palermo, Palermo, Italy. ${ }^{7}$ Laboratory of Physiology and Pharmacology, Université Libre de Bruxelles (Campus Erasme), Brussels, Belgium. ${ }^{8}$ Respiratory Physiology Unit, Cardiothoracic Dept, Cochin Hospital, Paris Descartes University, Paris, France. ${ }^{9}$ Pittsburgh Heart, Lung, Blood and Vascular Medicine Institute, Division of Pulmonary, Allergy and Critical Care Medicine, Dept of Medicine, University of Pittsburgh, Pittsburgh, PA, USA. ${ }^{10}$ Alfred Health, Monash University, Melbourne, Australia. ${ }^{11}$ Pulmonary and Critical Care Medicine, Medical School, National and Kapodistrian University of Athens, Athens, Greece. ${ }^{12}$ Dept of Pulmonology, AP-HP, Georges Pompidou European Hospital, Sorbonne Paris City, Medical School, Paris Descartes University, Paris, France. ${ }^{13}$ Dept of Hematology, Oncology and Molecular Medicine, Istituto Superiore di Sanità, Rome, Italy. ${ }^{14}$ Faculty of Medicine, Dept of Medicine, Imperial College London, London, UK. ${ }^{15} \mathrm{~A}$. Huertas and C. Guignabert have contributed equally. They are both first authors and are co-chairs of the European Respiratory Society (ERS) Research Seminar Faculty.
\end{abstract}

Correspondence: Alice Huertas, Inserm UMR_S 999, Hôpital Marie Lannelongue, 133 Avenue de la Resistance, 92350 Paris, France. E-mail: alice.huertas@inserm.fr

\section{@ERSpublications}

The central role of pulmonary endothelial dysfunction in respiratory diseases http://ow.ly/tVgK30iiZ1S

Cite this article as: Huertas A, Guignabert C, Barberà JA, et al. Pulmonary vascular endothelium: the orchestra conductor in respiratory diseases. Eur Respir J 2018; 51: 1700745 [https://doi.org/10.1183/ 13993003.00745-2017].

ABSTRACT The European Respiratory Society (ERS) Research Seminar entitled "Pulmonary vascular endothelium: orchestra conductor in respiratory diseases - highlights from basic research to therapy" brought together international experts in dysfunctional pulmonary endothelium, from basic science to translational medicine, to discuss several important aspects in acute and chronic lung diseases. This review will briefly sum up the different topics of discussion from this meeting which was held in Paris, France on October 27-28, 2016. It is important to consider that this paper does not address all aspects of endothelial dysfunction but focuses on specific themes such as: 1) the complex role of the pulmonary endothelium in orchestrating the host response in both health and disease (acute lung injury, chronic obstructive pulmonary disease, high-altitude pulmonary oedema and pulmonary hypertension); and 2) the potential value of dysfunctional pulmonary endothelium as a target for innovative therapies. 


\section{Introduction}

The lung is supplied by two distinct circulatory systems, the systemic bronchial circulation system and the pulmonary circulation system. From a functional point of view, the pulmonary circulation system is unique among vascular beds in the human body, characterised as it is by high-flow, low-resistance and low-pressure. Although all cardiac output is pumped through the pulmonary circulation system, the measured values of mean pulmonary arterial pressure (mPAP) in pulmonary arteries for a resting, healthy adult human are about $14 \pm 3 \mathrm{mmHg}$, whereas systemic arterial blood pressure is about $100 \pm 20 \mathrm{mmHg}$. This unique feature of the pulmonary circulation system is mainly explained by three critical characteristics of the pulmonary vasculature: 1) a low impedance to blood flow; 2) a high compliance of pulmonary precapillary arterioles characterised by a thin vessel wall; and 3) a high capacity to recruit available vessels that help to accommodate an increase in flow. Indeed, this circulatory system can accommodate flow rates ranging from approximately $6 \mathrm{~L} \cdot \mathrm{min}^{-1}$ under resting conditions to $25 \mathrm{~L} \cdot \mathrm{min}^{-1}$ under strenuous exercise, with minimal increases in pulmonary pressure [1]

Due to their strategic localisation at the interface between the bloodstream and lung tissue, pulmonary endothelial cells (ECs) play key roles not only in optimising gas exchange and in controlling barrier integrity and function, but also in regulating pulmonary vascular tone (i.e. through the nitric oxide (NO), prostacyclin $\left(\mathrm{PGI}_{2}\right)$, endothelin (ET) and serotonin (5-HT) pathways) [2]. Moreover, the pulmonary endothelium functions as an active and dynamic receptor-effector tissue and responds to different chemical, physical, or mechanical stimuli by secreting the correct substance(s) by which it may maintain vasomotor balance and vascular-tissue homeostasis. Indeed, pulmonary ECs are highly metabolically active, sensing and responding to signals from extracellular environments. All these interactions with adjacent cells (and circulating cells and/or mediators) aim to maintain a thrombosis-free surface, so as to control inflammatory cell adhesion and trafficking to assure normal angiogenesis and the integrity of the vascular wall [3]. As a vital part of the respiratory system, alteration of the pulmonary endothelium plays a central role in the pathogenesis of several chronic and acute lung diseases, both common and rare. The main characteristics of pulmonary endothelial alteration (or dysfunction) are increased permeability that can lead to vascular leakage and oedema formation; altered balance between vasoconstriction and vasodilation; acquisition of a pro-inflammatory phenotype with increased expression of adhesion molecules for inflammatory cell recruitment, increased activation of pro-inflammatory transcription factors, release of inflammatory mediators and oxidative stress; pro-thrombotic phenotype; pro-proliferative and anti-apoptotic phenotype; and miscommunication with adjacent vascular cell walls.

Although considerable progress has been made in the understanding of pulmonary EC dysfunction, the triggers, mechanisms and consequences of a dysfunctional endothelium in both acute and chronic lung disease are still not completely understood. A better knowledge of these key aspects would help in finding new disease biomarkers and/or novel therapeutic targets to preserve homeostasis in response to injury and disease.

\section{The importance of genetic and environmental factors}

Most, if not all, acute and chronic lung diseases result from interaction between multiple environmental exposures and many genetic risk factors, an aspect that has been critically evaluated in a recent review from the European Lung White Book [4]. The role of vasculature in the development, lifelong repair and maintenance of lung tissue homeostasis is of paramount importance. Indeed, many diseases involving the lung, such as emphysema, chronic obstructive pulmonary disease (COPD) [5, 6] and pulmonary hypertension (PH) [7], display major EC abnormalities. A better understanding of how pulmonary ECs contribute to normal lung development and how these mechanisms are deregulated during chronic disease is of crucial importance for the development of new therapeutic strategies for debilitating lung conditions. However, individual differences in subject response to the same stimulus, such as environmental hypoxia at high altitude, are well documented. In addition, it is well established that the pulmonary vasopressor response varies greatly between humans and animals (and even between different species), supporting the importance of unknown genetic influences.

Beyond the genetic background and specific gene mutations, other factors are known to determine structural and functional modifications to the pulmonary vasculature (such as exogenous exposure to drugs and toxins, hormones and aging), resulting in decreasing repair capacity [8-10] and impaired production of different vasoreactive mediators [11].

Another potential agent that is capable of causing endothelial impairment is cigarette smoke (tobacco products), which play a major role in COPD, since "healthy smokers" (without COPD) already show significant abnormalities in their pulmonary arteries including: vessel remodelling, inflammatory cell infiltrate, endothelial dysfunction, reduced expression of endothelial NO synthase (eNOS), increased expression of growth factors and a similar gene expression profile to that observed in COPD patients [12, 13]. 
It has also been demonstrated in experimental models of COPD that chronic exposure to cigarette smoke induces endothelial dysfunction in pulmonary arteries [14], along with changes in the expression of eNOS [15] and soluble guanylate cyclase (sGC) [16]. Interestingly, vascular changes precede the development of pulmonary emphysema [17]. Altogether, these findings strongly support the relationship between smoking and pulmonary vascular damage in COPD.

\section{Properties of dysfunction of the endothelial barrier}

The vascular endothelium forming the innermost lining of all pulmonary blood vessels is the major barrier that protects air spaces against vascular fluid entry. The integrity of the permeability of this endothelial barrier is critical for the maintenance of homeostasis for all the tissues and organs of the body and changes in its permeability are a hallmark of acute and chronic lung disease.

The vascular endothelium forms a continuous uninterrupted layer of ECs that is held together by complex junctional structures, namely adherens junctions, tight junctions and gap junctions, which are known as gate keepers for trans-endothelial movements of fluid, protein and cells. It is well established that endothelial barrier permeability under basal conditions and in response to different stimuli or certain agents, varies considerably among the different vascular beds [18]. Loss of the endothelial barrier causes pulmonary oedema, the characteristic feature of acute lung injury (ALI), as well as high-altitude pulmonary oedema (HAPE). The pathogenesis of both conditions is still unclear and no drugs have been approved so far for these life-threatening conditions.

ALI can progress to acute respiratory distress syndrome (ARDS) in which multi-organ failure accounts for a high degree of mortality and morbidity. ARDS can be precipitated by either direct insults to the lung (i.e. pneumonia or aspiration of gastric contents) or indirect insults (i.e. sepsis or multiple trauma). Sepsis is the most common cause of ARDS in humans and it is also well-established that a pulmonary cause carries with it the highest mortality compared with other ARDS aetiologies. The incidence of ARDS varies widely, from 15 to 70 cases per 100000 persons per year, representing approximately 5\% of hospitalised, mechanically ventilated patients [19].

Being at the interface between the vascular and lung airspaces, the pulmonary endothelium is positioned to play a critical role in establishing immune responses that lead to ALI. The mechanistic understanding underlying these processes remains inadequate and is considered here in light of new findings. The lung's innate immune response involves recruitment of immune cells to pulmonary vessels across which they migrate to the air space. Injury to the endothelial barrier occurs through the paracrine effect of mediators (such as arachidonate [20], ATP [21] and peroxide [22]) released from adjoining epithelial cells, mechanical stresses such as vascular stretch resulting from increased vascular pressure [23], peroxide release from hypoxic red cells [24] and pro-coagulant proteins deposed by platelets [25].

A surprising yet robust role for endothelial mitochondria in the lung's immune response is becoming increasingly evident, even though mitochondria are generally considered to be major sites of cellular ATP generation and little is known about the functional significance of lung endothelial mitochondria in the context of the normal or the diseased lung. The old impression that ECs lack mitochondria, as derived from cultured cells, has been dispelled by the more recent real-time fluorescence imaging (RFI) of live lungs in studies that provide definitive evidence for the presence of lung endothelial mitochondria [26]. As a result of enhanced cytosolic $\mathrm{Ca}^{2+}$ oscillation, either spontaneous [27] or induced by conditions associated with ALI $[26,28]$, oscillation of mitochondrial $\mathrm{Ca}^{2+}$ increases causing mitochondrial hydrogen peroxide production and therefore nuclear factor $-\kappa B(N F-\kappa B)$ pathway activation $[26,28]$. The resulting gene transcription leads to endothelial expression of P-selectin and leukocyte adhesion receptor E-selectin, the defining marker of pro-inflammatory activation in the lung vasculature. In the pulmonary endothelium, mitochondrial density tends to be somewhat higher at capillary branch points than in the septal capillaries [23]. As such, inflammation initiates at capillary branch points, which are also major sites of microvascular filtration, a process which generates the interstitial fluid required to drive lymph flow. Thus, lung endothelial mitochondria activate innate immune mechanisms that in turn induce physiological responses to facilitate lymphatic delivery of antigens to lymph nodes, promoting adaptive immunity (figure 1).

A protective role for endothelial mitochondria has also emerged through RFI studies addressing the expression of an endothelial pro-inflammatory receptor, tumour necrosis factor (TNF) receptor 1 (TNFR1), which is expressed as a trans-membrane protein on the luminal membrane [28]. The mitochondrial release of hydrogen peroxide resulting from tumour necrosis factor- $\alpha$ (TNF- $\alpha$ ) induced $\mathrm{Ca}^{2+}$ oscillations activates a metalloproteinase, TNF- $\alpha$ converting enzyme (TACE), that is also expressed on the luminal endothelial membrane, causing shedding of TNFR1 ectodomains and limiting the pro-inflammatory effects of TNF- $\alpha$. 
FIGURE 1 Inflammation and pulmonary microcirculation. Inflammation-mediated events occurring in pulmonary arterioles, capillaries and venules contribute to impaired capillary perfusion. The sample presented was taken from the lungs of a patient with chronic thromboembolic pulmonary hypertension. Note the moderate to severe microvascular inflammation (in the absence of any major vascular wall remodelling). The scale bar represents $100 \mu \mathrm{m}$.

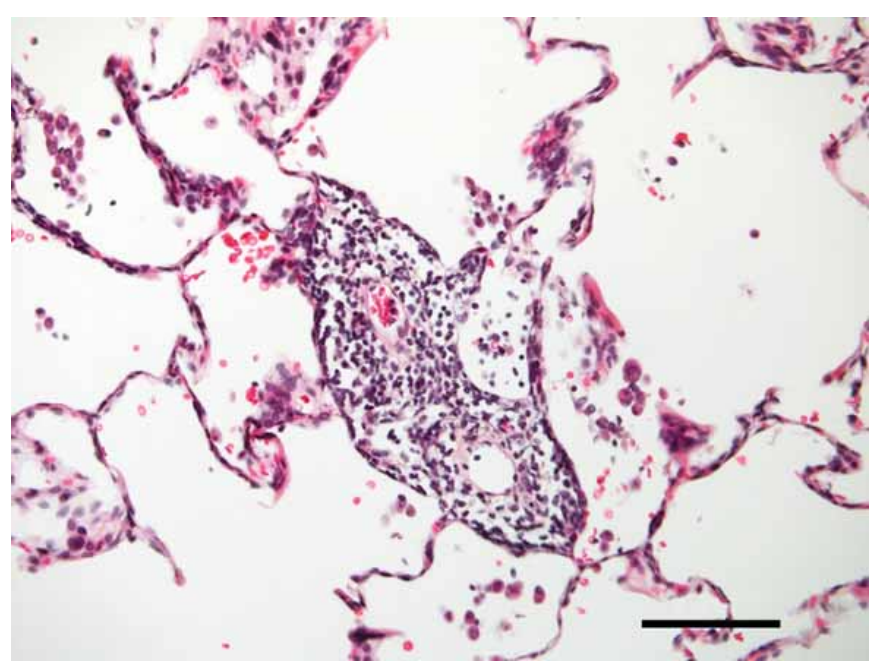

The new understanding that mitochondria are immune sensors may have implications beyond the pathology of ALI to inflammatory lung diseases in general [29]. Further research is required to understand disease processes that damage lung mitochondria, particularly in the pulmonary endothelium and to develop therapeutic strategies for improving mitochondrial function to cure lung diseases.

Loss of endothelial barrier properties in the pulmonary circulation system can also cause a high-permeability form of pulmonary oedema, defined as HAPE, that can occur in previously healthy, fit, but not acclimatised individuals who ascend rapidly to altitudes above $3000-4000 \mathrm{~m}$ [30, 31]. It is a multifactorial disease involving both environmental (altitude and rate of ascent) and genetic risk factors [32-34]. In addition, oxygen deprivation causes vasoconstriction of the small pulmonary arteries when systemic arteries dilate under hypoxic conditions. This phenomenon, known as hypoxic pulmonary vasoconstriction (HPV), is more pronounced as the vessel diameter decreases and represents an important physiological mechanism by which pulmonary arteries constrict in hypoxic lung areas to redirect blood flow to areas with greater oxygen supply. It is now well recognised that chronic exposure to hypoxia (i.e. COPD or living at high altitude) causes continuous alveolar hypoxia, resulting in sustained HPV that is thought to play a crucial role by exposing the pulmonary capillaries to high pressure, damaging their walls and leading to pulmonary vascular remodelling, and possibly to $\mathrm{PH}[35,36]$.

Abnormally high pulmonary arterial pressure (PAP) in hypoxia has been shown to be a hallmark of HAPE. HAPE susceptibility can be revealed at low altitude during a brief hypoxic challenge in people with a history of HAPE [37-39]. Right-heart catheterisation performed at high altitude showed that all individuals developing pulmonary oedema had capillary pressures above a leakage threshold of $19 \mathrm{mmHg}$, indicating that abnormally high PAP in HAPE translates to the capillary bed [40-42]. Areas with less HPV have higher perfusion causing flow-mediated increased capillary pressure and leakage, while areas with more vigorous vasoconstriction have lower perfusion and are thus protected from oedema formation. This hypothesis is supported by perfusion scan results in HAPE which show that pulmonary oedema occurs in areas of high blood flow [31], the observation that oedema has a patchy distribution in HAPE [43] and by analysis of lung perfusion in hypoxia by magnetic resonance imaging (MRI) [44, 45]. In addition, bronchoalveolar lavage (BAL) performed at high altitude in individuals at the outset of HAPE reported an absence of inflammatory markers in the BAL fluid [46]. However, inflammatory cells and cytokines were found in BAL fluid in more advanced cases [47] and in patients hospitalised with HAPE in Japan [48]. HAPE is thus a pressure-induced noninflammatory oedema that may induce a secondary inflammatory response [30, 49].

A recent meta-analysis of high-altitude studies, in which PAP was measured by echocardiography in people living at altitudes between 3600 and $4350 \mathrm{~m}$, concluded that altitude-induced $\mathrm{PH}$ appears to be rare [50]. Mean systolic PAP at high altitude was $25.3 \mathrm{mmHg}$, which was modestly elevated compared to that seen in healthy individuals from the general population living below $1200 \mathrm{~m}$. Nonetheless, there is a wide distribution and some people are protected from $\mathrm{PH}$ at altitude compared to others [51]. Others have reported that the magnitude of HPV in humans can vary almost five-fold between individuals [52, 53]. This has a genetic basis and understanding the genetic mechanisms involved offers important insights into the molecular regulators of pulmonary vascular tone and structure which can be exploited as drug targets. Studies examining extreme phenotypes in high-altitude populations and rat strains have begun to support this approach and open up new areas of research. 
HAPE-susceptible individuals display endothelial dysfunction in forearm blood flow studies in hypoxia but not in normoxia, reduced exhaled NO at high altitude $[54,55]$, decreased pulmonary NO bioavailability $[31,46]$ and an increase in production of reactive oxygen species (ROS) across the lung (particularly at the pulmonary artery smooth muscle cell (PA-SMC) level in hypoxia) [56]. In addition, plasma levels of endothelin-1 (ET-1) increase in parallel with increased PAP in HAPE-susceptible individuals at high altitude [57]. Besides these endothelium-dependent mechanisms accounting for increased HPV in HAPE-susceptible individuals, additional factors must be considered such as a low hypoxic ventilatory response [58] and a smaller capillary bed as suggested by lower lung volumes [59, 60], which can both contribute to increased PAP in hypoxia.

There is a need to better understand the mechanisms underlying the differences in endothelial permeability of various vascular beds, the different signalling pathways that modulate inter-endothelial junctions and how tissue-fluid homeostasis is controlled under normal conditions and in pathological processes. Indeed, the restoration of the endothelial barrier is critical for tissue homeostasis and also for recovery following exposure to acute inflammatory events or injury. However, our understanding of how this process occurs at the molecular level remains obscure. More effort should be geared toward developing drugs that facilitate the formation of inter-endothelial junctions or that enhance the function of key mediators of barrier integrity.

\section{Altered balance between vasoconstriction and vasodilation}

As already underlined, the pulmonary circulation system has unique haemodynamic features, characterised by a more relaxed vasomotor tone in the normal physiological state, in contrast to the systemic circulation system. In the fetus, where gas exchange occurs in the placenta, HPV acts to maintain high pulmonary vascular resistance and to divert blood flow away from the lungs through the ductus arteriosus. Different ion channels, including potassium $\left(\mathrm{K}^{+}\right)$channels, are expressed and distributed in several types of pulmonary vascular cells (including PA-SMCs) and contribute to the large functional diversity in the hypoxic response.

Pulmonary ECs synthesise a variety of paracrine and endocrine factors that are capable of controlling vascular tone, either by favouring relaxation (i.e. $\mathrm{PGI}_{2}$ and $\mathrm{NO}$ ) or by causing contraction (i.e. ET-1 and 5-HT) under basal conditions and in response to different stimuli or certain agents [61]. There is evidence to suggest that background release of NO contributes to the normally low pulmonary vascular tone in normoxia [61]. Although there are theoretical grounds to hypothesise that hypoxia reduces the synthesis of $\mathrm{NO}$, lack of the latter does not seem to account for the acute HPV. Instead, there is evidence to suggest that NO activity is increased in order to modulate the pulmonary vasopressor response to acute alveolar hypoxia. The impaired NO production, whilst reducing the ability of the pulmonary vasculature to relax, also favours the occurrence of excessive pulmonary vasoconstriction [5]. Lack of NO synthesis might also permit mitogenesis and proliferation of various cell types within the vascular wall [62]. It is also well established that the RhoA/Rho-associated kinase (ROCK) pathway also regulates pulmonary vascular tone through both NO-dependent and NO-independent mechanisms [63]. Hypoxia-induced activation of ROCK pathways increases the $\mathrm{Ca}^{2+}$ sensitivity of the contractile myofilaments in PA-SMCs, thereby increasing vascular tone and causing sustained HPV and increased pulmonary vascular resistance [63-65].

In the adult, HPV has at least two phases: the initial constrictor response starts within seconds and reaches a maximum within minutes, followed after 30-120 min by a sustained phase [52, 64, 66]. These phases are regulated, at least in part, by different signalling pathways. The second phase of HPV is influenced by EC function through changes in the release of vasoactive mediators such as ET-1, $\mathrm{PGI}_{2}$ and NO. In vivo, neurohumoral mediators, red blood cells and lung innervation may also influence the response. The mechanisms underlying the sensing of low oxygen levels by cells have been the subject of discussion. Both mitochondria and NAHD/NADPH oxidases have been suggested as oxygen sensors. A change in the levels of ROS is thought to be important but there is a lack of agreement about whether the signal is an increase or decrease in ROS. Hypoxia-inducible factor 1 (HIF-1) and HIF-2 that transactivate a number of common as well as distinct downstream target genes are clearly candidates for explaining variability in the pulmonary vascular response to chronic hypoxia [67]. In addition to differing in terms of downstream targets, their $\alpha$-subunit expression levels are also known to vary in cells and tissues. Recently, a comparison of two rat strains, F344 and WKY, which differ in their response to chronic hypoxia, has highlighted the importance of zinc in several cell types, particular PA-SMCs, in regulating pulmonary vascular homeostasis [68]. A mutation in the solute carrier family 39 member 12 gene (Slc39a12), which encodes the zinc transporter ZIP12, predicts a truncated and inactive protein in the F344 rat strain. Introduction of a similar mutation in the WKY rat strain reduces its responsiveness to hypoxia. Upregulation of ZIP12 expression in the lungs of humans living at high altitude (and patients with idiopathic pulmonary arterial hypertension (PAH)) has now stimulated a search for small molecule inhibitors of ZIP12, as well as interest in the role of intracellular labile zinc in pulmonary vascular disease. 


\section{From dynamic to unadapted pulmonary vascular remodelling}

The pulmonary circulation system responds to alveolar hypoxia by increasing pulmonary vascular resistance and HPV is responsible for the initial rise in pulmonary pressures $[4,52,53]$. However, with continued exposure to hypoxia, other mechanisms drive structural changes in resistance vessels which contribute to the elevated pressures, thus explaining why after 2 or 3 weeks of hypoxia there is little response to rebreathing $100 \%$ oxygen. Notably, pulmonary pressures decrease progressively with time on re-exposure to a normal oxygen environment, suggesting that the pulmonary vascular remodelling induced by chronic hypoxia is reversible. Histological examination of lungs from mammals, including rats, cows and humans exposed chronically to hypoxia, demonstrate structural remodelling of pulmonary arterioles [69]. All layers of the vascular wall are involved in the remodelling, including fibroblasts, but the hallmark of the vascular response to chronic hypoxia is increased muscularisation of distal vessels with extension of muscle into previously non-muscularised arterioles (figure 2). The relative contributions of HPV and vascular remodelling to chronic hypoxia-induced $\mathrm{PH}$ are the subject of debate [70]. Histological studies in rats contest the extent to which remodelling narrows vascular lumen and provides an obstruction. A recent consideration in this debate is vascular stiffness, where the changes in vascular structure lead to an increase in stiffness. This stiffness alters the transmission of pulse waves along affected vessels and their reflection from branch points and the right heart then senses an increased pressure load from the combination of advancing and reflected waves. The role of haemodynamic forces and shear stress in initiating and sustaining pulmonary vascular remodelling in hypoxia has been highlighted recently by studies in rats. Pulmonary artery banding reduces haemodynamic shear stress and not only prevents the development of vascular remodelling but also reverses occlusive lesions and perivascular inflammation in the Sugen hypoxia model [71]. It is easy to speculate that haemodynamic stress and occlusive vascular lesions could form a vicious cycle. If so, these observations suggest that mechanisms to reduce shear stress, for example with vasodilator therapies, should not be ignored in the search for treatments for $\mathrm{PH}$.

Defined as mPAP $\geqslant 25 \mathrm{mmHg}$ at rest as assessed by right-heart catheterisation, $\mathrm{PH}$ can present in a number of clinical scenarios including several with a high mortality rate [72, 73], as follows: PAH (group 1); $\mathrm{PH}$ due to lung diseases such as COPD and emphysema and/or hypoxia (group 3); chronic thromboembolic pulmonary hypertension (CTEPH) (group 4); $\mathrm{PH}$ with unclear multifactorial mechanisms, such as haematological disorders (group 5) [74]. In all of these PH subgroups, pulmonary endothelial dysfunction contributes to pulmonary vascular remodelling $[75,76]$. In particular, major functional alterations in the pulmonary vascular endothelium have been demonstrated in PAH, including among others: 1) a transition from a quiescent state without adhesive capacity to an activated state with adhesive capacity [77]; 2) an aberrant pro-proliferative and apoptosis-resistant phenotype [78]; 3) a pro-inflammatory phenotype characterised by an excessive release of various key cytokines and chemokines such as interleukin (IL)-1 $\alpha$, IL-6, IL-8, IL-12 and chemokine (C-C motif) ligand 2 (CCL2)/ monocyte chemotactic protein-1 (MCP-1) [77]; and 4) an excessive production and secretion of various key growth factors including fibroblast growth factor-2 (FGF-2) $[78,79]$, angiotensin (Ang II) [80] and leptin [81-83]. Additional insights into the altered pulmonary EC phenotype and endothelial communication with both resident vascular cells (i.e. PA-SMCs and myofibroblasts) and immune cells are a prerequisite for a better understanding of $\mathrm{PAH}$ pathogenesis that could lead to novel therapeutic strategies.
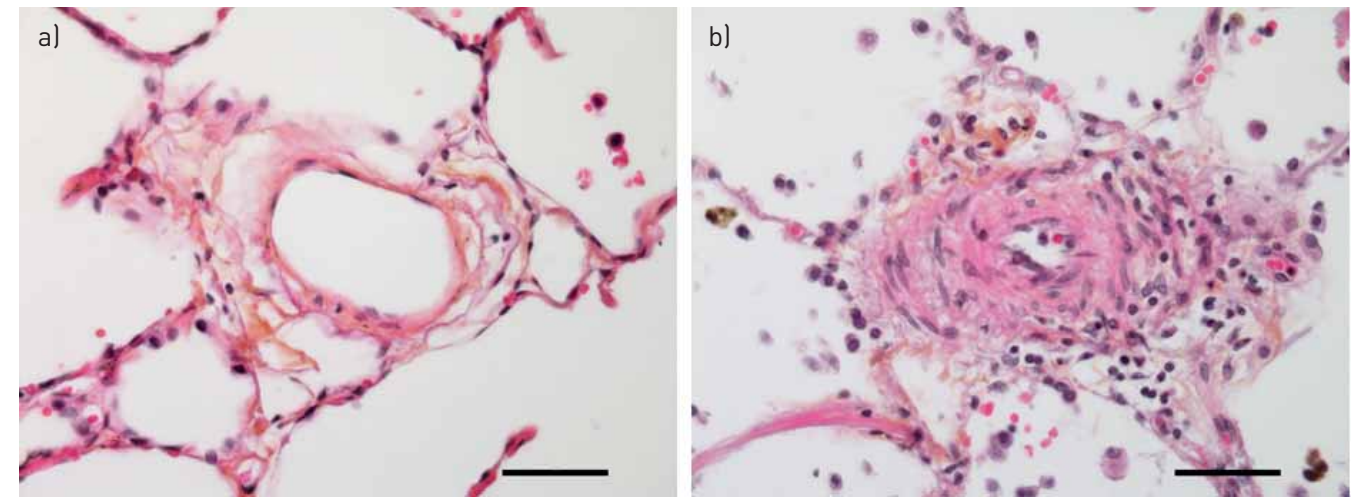

FIGURE 2 Vascular response to chronic hypoxia. Previously nonmuscularised arterioles (a) show "muscularisation" with recruitment of smooth muscle cells and myofibroblasts (b). Note the mild inflammatory infiltrate (predominating on the right side of the vessel) which accompanies the remodelling process. The scale bars represent $50 \mu \mathrm{m}$. 
More recently, endothelial communication was reported with pericytes, a key type of progenitor cell, which are found around pre-capillary arteries, capillaries and post-capillary venules. They also occupy a strategic position at the interface between circulating blood and interstitial space, and in close proximity to ECs and PA-SMCs. RICARD et al. [84] have demonstrated excessive pericyte coverage (a two to three-fold increase) in distal pulmonary arteries in human $\mathrm{PAH}$, a phenomenon that contributes to pulmonary vascular remodelling as a source of smooth-muscle-like cells. Pericytes are central regulators of vascular development, stabilisation and maturation, as well as modulation of remodelling by affecting: 1) EC growth, proliferation, differentiation and migration; 2) PA-SMC contraction; 3) immune cell function; 4) acting as progenitor cells and possible differentiation in SMC-like cells and dendritic cells.

Deciphering the intracellular pathways that modulate pulmonary vascular remodelling has paved the way for precise molecular targeting strategies in new treatments for $\mathrm{PH}$ [85]. For example, one can now choose between specific and nonspecific ET-1 receptors, or stimulation of synthesis or inhibition of degradation of the key intracellular second messenger, cyclic guanosine monophosphate (cGMP), to alter pulmonary vascular tone in diseased states. Even though the prognosis remains poor, these additional pathways may represent innovative tools for the treatment of $\mathrm{PH}$ in the future.

In $\mathrm{PH}$ due to a lung disease, such as COPD, pulmonary vascular impairment is highly prevalent with about $40 \%$ of patients with Global Initiative for Chronic Obstructive Lung Disease (GOLD) Stage 4 COPD and $27 \%$ of patients with GOLD Stage 3 COPD displaying an increased mPAP [86]. Among patients without $\mathrm{PH}$ when at rest, a significant proportion develop it during exercise [87, 88]. These findings concur with the frequent observation of histological changes, mainly consisting of intimal hyperplasia, in the small pulmonary arteries in patients with end-stage disease who underwent lung transplantation [5], even in patients with mild-to-moderate disease $[6,89]$. In addition to vascular remodelling, dysregulated vascular tone is a major contributor to $\mathrm{PH}$ in COPD: the expression of eNOS [90], sGC [16] and $\mathrm{PGI}_{2}$ synthase [36] are reduced in the pulmonary arteries of COPD patients, thus decreasing their vasodilator and antiproliferative capacity (figure 3).

With this background, it is interesting to note that some patients with extensive emphysema develop severe $\mathrm{PH}$ [91], suggesting a link between the emphysematous destruction of lung parenchyma and the severity of pulmonary vascular impairment. In fact, in explanted lungs of COPD patients who underwent lung transplant, the severity of emphysema was significantly higher in those patients who had PH [92]. Accordingly, the combination of vascular remodelling, endothelial dysfunction and parenchymal derangement might explain the development of severe $\mathrm{PH}$ in a subset of patients with COPD [93].

The pulmonary endothelium represents an interesting therapeutic target in COPD. Studies conducted in experimental models show that targeting the NO-cGMP signalling pathway with sGC stimulators [16] or phosphodiesterase-5 (PDE5) inhibitors [94, 95] prevents the development of $\mathrm{PH}$, pulmonary vascular remodelling and right-ventricle hypertrophy. Interestingly, sGC stimulators and PDE5 inhibitors also attenuated the development of pulmonary emphysema [16, 95], an effect that was associated with a reduction in the number of neutrophils and alveolar macrophages in lung tissue, as well as a reduction in leukocyte adhesion [16]. Taken together, these findings suggest that therapeutic interventions in the NOcGMP signalling pathway exert a dual effect: 1) relaxation and eventually attenuation of cell proliferation

FIGURE 3 A pulmonary artery in a patient with chronic obstructive pulmonary disease. The vessel lshown here at a dichotomous branching point) presents with important intimal fibrosis. Note the mild perivascular infiltrate (predominating beneath the artery). The surrounding pulmonary parenchyma displays emphysematous remodelling. The scale bar represents $200 \mu \mathrm{m}$.

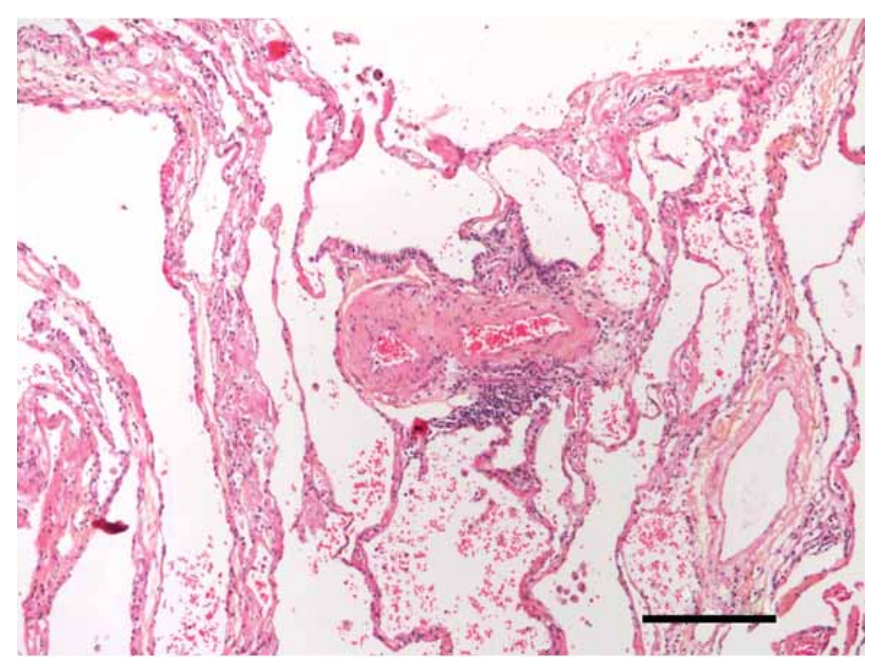


through protein kinase $G$ dependent mechanisms in smooth muscle cells; 2) reduction of leukocyte adhesion in ECs, presumably by downregulating P-selectin [96].

These results have been obtained using a "preventive experimental design" but it remains to be shown as to what extent the therapeutic intervention exerts the same effects when lung damage is already established (i.e. using a "therapeutic design"). In fact, treatment of COPD patients with PH using PDE5 inhibitors or sGC stimulators has been ineffective in improving exercise tolerance [86]. However, exercise tolerance might not be the most adequate surrogate for the effects of treatment on the vascular endothelium. Further experimental and clinical studies addressing the effects of therapeutic interventions on the disturbed endothelium are warranted to elucidate the significance of this approach in COPD.

In PH due to hematological disorders, such sickle cell disease (SCD) [97-101], the mechanistic linkage between haemolytic anaemia and vasculopathy has been the subject of extensive study in pre-clinical animal models and in vascular studies in patients, as well as in large human cohort studies [99, 102, 103]. Intravascular haemolysis releases cell-free haemoglobin $(\mathrm{Hb})$ in the plasma that can scavenge $\mathrm{NO}$ and generate ROS, impairing redox balance and leading to proliferative systemic and pulmonary vasculopathy $[103,104]$. It has recently been noted that products released from red cells during haemolysis can be considered as danger-associated molecular patterns (DAMPs) or erythrocyte DAMPs (eDAMPs) [105]. These studies in SCD support a more general pathological role for intravascular haemolysis and cell-free $\mathrm{Hb}$ in various human diseases and in transfusion medicine [106].

\section{Biological factors to assess pulmonary endothelial dysfunction}

In light of the critical role played by the pulmonary endothelium in acute and chronic lung diseases, identification of biological factors to assess its (dys)function in the field is needed; however, this represents a real challenge for clinicians and researchers. Indeed, a broad appreciation of the numerous functions of the endothelium requires the assessment of a large panel of molecules of endothelial origin in circulating blood (table 1) and comprehensive prior assessment and validation in large and well-characterised cohorts of patients before routine clinical use. These EC products could include, among others: measures of NO biology, circulating miRNAs, cytokines, chemokines, circulating adhesion molecules, growth factors and

TABLE 1 A partial list of endothelium-derived pulmonary factors

Pulmonary factor $\quad$ Type

Vasoconstrictors

Vasodilators

Cell accumulation

Inflammation

Other proteins
Type

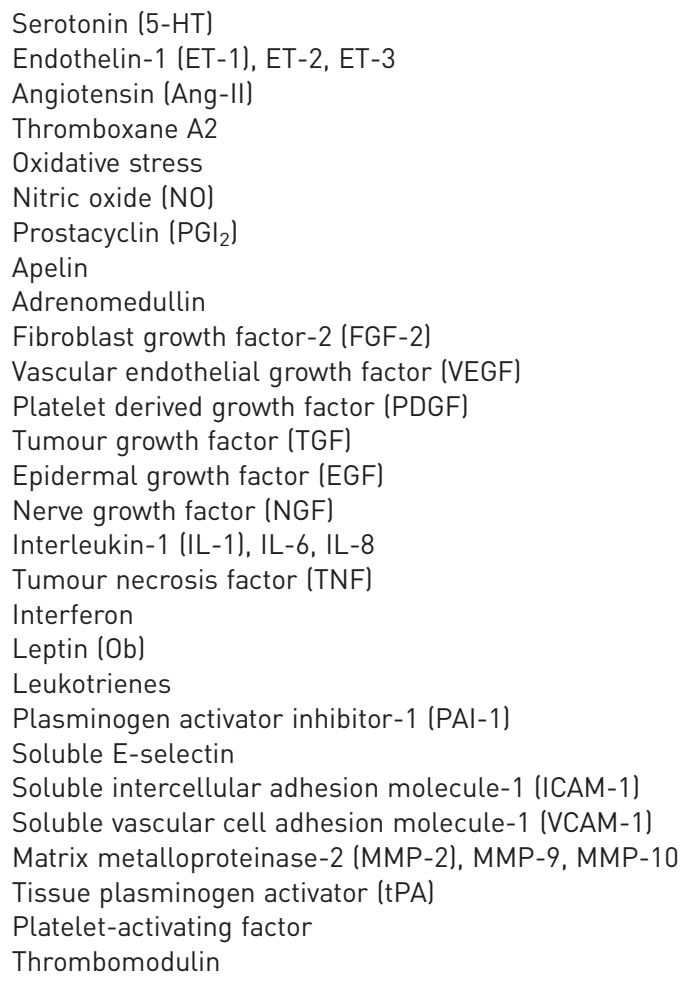




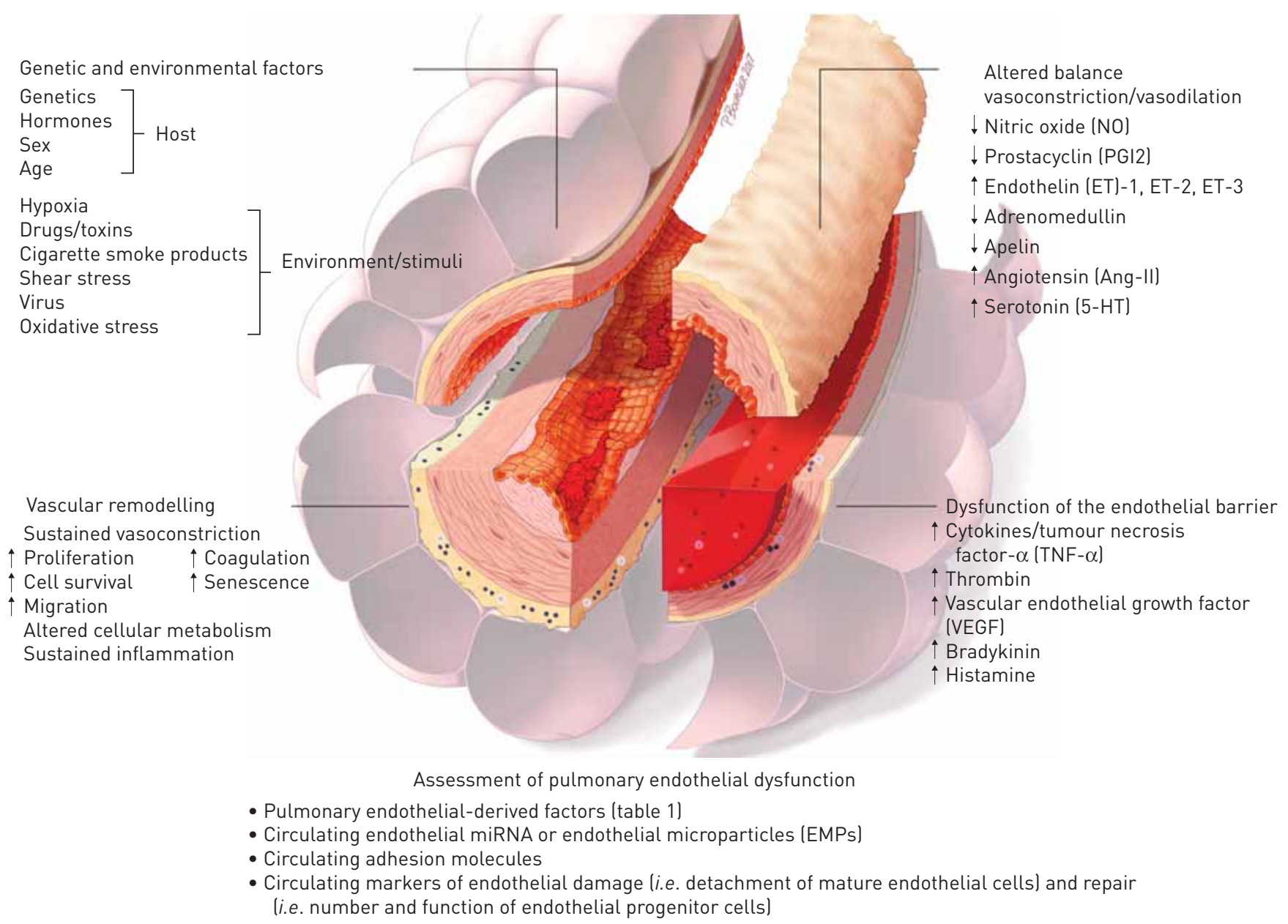

FIGURE 4 A schematic overview of the main characteristics of pulmonary endothelial dysfunction.

regulators of thrombosis, as well as markers of endothelial damage (i.e. detachment of mature ECs or derived endothelial microparticles (EMPs)) and endothelial repair (i.e. the number and functional characteristics of circulating endothelial progenitor cells).

Besides changes in vasoactive mediators, COPD patients present with structural damage in the pulmonary endothelium. Areas of cell denudation and detachment between ECs have been shown in the pulmonary arteries of COPD subjects [107]. The presence of endothelial damage in COPD has also been demonstrated by increased numbers of EMPs [108, 109]. Circulating EMPs may originate from EC apoptosis or activation, which can be identified by the expression of the CD31 and CD62E markers, respectively. The number of circulating EMPs is inversely related to the forced expiratory volume in $1 \mathrm{~s}$ [109]. Interestingly, whereas CD31 EMPs (apoptotic) are already significantly elevated in patients with mild COPD, CD62E EMPs (activated) are increased only in patients with severe disease. The number of circulating EMPs further increases during exacerbation episodes [110] and also correlates with the severity of emphysema [109], pointing to a connection between parenchymal destruction and endothelial damage. Furthermore, in COPD, circulating EMPs are also correlated with the vascular function of systemic arteries. An increased number of circulating EMPs is associated with impaired endothelial function and increased stiffness in systemic arteries [111]. COPD patients also show reduced numbers of bone marrow derived circulating endothelial progenitor cells (EPCs) [112-115], which play a key role in the repair of a damaged endothelium and are closely related to endothelial function [116]. Studies carried out during the last years have made great effort to try and identify, define and characterise cell populations with phenotypic, biochemical, molecular and functional properties of EPCs [117, 118]. EPCs have been suggested as a precious source for generating functionally competent ECs, candidates for various clinical applications. However, the paucity of these progenitor cells and the technical difficulties involved in their in vitro growth represent a significant limitation to their use. Interestingly, recent studies have led to the identification in haematopoietic tissues (blood, bone marrow and cord blood) of very rare EPCs not 
originating from bone marrow. These endothelial colony-forming cells (ECFCs) or "late outgrowth ECs" are capable of generating a large progeny of phenotypically and functionally competent mature ECs in vitro and also of sustaining in vivo angiogenetic processes $[119,120]$. Such EPCs are also present at the level of the vessel wall, particularly in the vascular endothelial intima $[117,118]$. This methodology can be easily extended to the study of circulating EPCs and can facilitate and improve the functional characterisation of EPCs in various pathologic conditions, particularly in various lung diseases.

\section{Conclusion}

In conclusion, it is now well established that the pulmonary vascular endothelium is involved in most if not all acute and chronic lung disease, either as a primary determinant of these disease processes or as a victim of collateral damage (figure 4). Although considerable progress has been made in the understanding of pulmonary EC (dys)function, the triggers, mechanisms and consequences of a dysfunctional endothelium in lung disease, both acute and chronic, are still not completely understood. In addition, further efforts are also needed to elucidate the remarkable heterogeneity of the pulmonary endothelium in space and time, in structure and function, and in health and disease. An understanding of the molecular basis for these key aspects would help in finding new disease biomarkers and/or novel therapeutic targets to preserve homeostasis in response to injury and disease and to reduce the "bench-to-bedside" gap in endothelial biomedicine.

Acknowledgements: The organisers of the European Respiratory Society (ERS) Research Seminar thank the faculty members, the chairs, the ERS scientific activities assistants and all the participants: A. Abd Al-Aziz (Cairo, Egypt), E. Barreiro (Barcelona, Spain), H. Bendjenana (Paris, France), I. Benhamou-Tarallo (Paris, France), O. Bernard (Paris, France), I. Bonovolias (Thessaloniki, Greece), J. Bordenave (Paris, France), I. Campean (Medias, Romania), P. Dybantsa (Paris, France), S. Fairhall (Salisbury, UK), M. Felten (Berlin, Germany), P. Ferreira (Porto, Portugal), N. Frossard (Illkirch, France), C. Guibert (Bordeaux, France), B. Jugg (Salisbury, UK), I. Khachatryan (Yerevan, Armenia), M. Kontic (Belgrade, Serbia), K.B. Kurakula (Amsterdam, The Netherlands), A.K. Larsson-Callerfelt (Lund, Sweden), E. Letsiou (Berlin, Germany), N. Mesropyan (Yerevan, Armenia), V. Michel (Bordeaux, France), R. Palma (São Paulo, Brazil), C. Phan (Paris, France), E. Rossi (Paris, France), K. Samara (Athens, Greece), D. Santos Ribeiro (Brussels, Belgique), S. Schmitt-Grohé (Bonn, Germany), A. Sekine (Chiba, Japan), V. Smolders (Barcelona, Spain), R. Szulcek (Amsterdam, The Netherlands), Y. Taniguchi (Kobe, Japan), R. Thuillet (Paris, France), L. Tu (Paris, France), O. Tura Ceide (Barcelona, Spain), J. Weatherald (Calgary, Canada).

Conflict of interest: J.A. Barberà has received personal fees (for acting on advisory boards, consulting and speaking) from Bayer, Actelion and GlaxoSmithKline, and institutional grants from Bayer, Actelion, GlaxoSmithKline and Pfizer, outside the submitted work. P. Bärtsch has received personal fees for lectures from Novartis, Permamed AG, MSD and Mundi Pharma, and personal fees for consultancy from Bayer AG, outside the submitted work. P. Dorfmüller has received personal fees from MSD and Actelion, outside the submitted work. M.T. Gladwin has a nitrite-related patent broadly relevant to pulmonary hypertension with royalties paid. M. Humbert has relationships with drug companies including Actelion, Bayer, GlaxoSmithKline, Novartis and Pfizer. In addition to being an investigator in trials involving these companies, relationships include consultancy service and membership of scientific advisory boards. O. Sanchez has received personal fees from BMS, grants, personal fees and nonfinancial support from MSD, Actelion and Bayer, grants from Portola and Daiichi Sankyo, and personal fees and nonfinancial support from Chiesi, outside the submitted work. L. Savale has received grants, personal fees and nonfinancial support from Actelion, MSD and Bayer, and grants and nonfinancial support from GlaxoSmithKline, outside the submitted work. M.R. Wilkins has a patent ZIP12 as a therapeutic target pending.

Support Statement: The research seminar was sponsored by the European Respiratory Society (ERS) with the financial support of unrestricted grants from Association de Recherche en Physiopathologie Respiratoire, Elivie, GlaxoSmithKline, Miltenyi Biotec, Oxyvie, Teva and Vivisol. Funding information for this article has been deposited with the Crossref Funder Registry.

\section{References}

West JB. Blood flow to the lung and gas exchange. Anesthesiology 1974; 41: 124-138.

Ryan US, Ryan JW. Cell biology of pulmonary endothelium. Circulation 1984; 70: III46-III62.

Ryan US. Pulmonary endothelium: a dynamic interface. Clin Invest Med 1986; 9: 124-132.

Gibson GJ, Loddenkemper R, Lundback B, et al. Respiratory health and disease in Europe: the new European Lung White Book. Eur Respir J 2013; 42: 559-563.

5 Dinh-Xuan AT, Higenbottam TW, Clelland CA, et al. Impairment of endothelium-dependent pulmonary-artery relaxation in chronic obstructive lung disease. N Engl J Med 1991; 324: 1539-1547.

6 Peinado VI, Barbera JA, Ramirez J, et al. Endothelial dysfunction in pulmonary arteries of patients with mild COPD. Am J Physiol 1998; 274: L908-L913.

7 Guignabert C, Dorfmuller P. Pathology and pathobiology of pulmonary hypertension. Semin Respir Crit Care Med 2013; 34: 551-559.

8 Marijic J, Li Q, Song M, et al. Decreased expression of voltage- and $\mathrm{Ca}^{2+}$-activated $\mathrm{K}^{+}$channels in coronary smooth muscle during aging. Circ Res 2001; 88: 210-216.

9 Herrera MD, Mingorance C, Rodriguez-Rodriguez R, et al. Endothelial dysfunction and aging: an update. Ageing Res Rev 2010; 9: 142-152.

10 Hoffmann J, Haendeler J, Aicher A, et al. Aging enhances the sensitivity of endothelial cells toward apoptotic stimuli: important role of nitric oxide. Circ Res 2001; 89: 709-715. 

Med 2013; 65: 380-401.

12 moderate COPD. Pulm Pharmacol Ther 2011; 24: 32-41. the guinea pig. Respir Res 2009; 10: 76. pulmonary hypertension and emphysema. Am J Respir Crit Care Med 2014; 189: 1359-1373. Seimetz M, Parajuli N, Pichl A, et al. Inducible NOS inhibition reverses tobacco-smoke-induced emphysema and pulmonary hypertension in mice. Cell 2011; 147: 293-305.

18 Mehta D, Bhattacharya J, Matthay MA, et al. Integrated control of lung fluid balance. Am J Physiol Lung Cell Mol Physiol 2004; 287: L1081-L1090.

19 Sweatt AJ, Levitt JE. Evolving epidemiology and definitions of the acute respiratory distress syndrome and early acute lung injury. Clin Chest Med 2014; 35: 609-624.

20 Kuebler WM, Parthasarathi K, Wang PM, et al. A novel signaling mechanism between gas and blood compartments of the lung. J Clin Invest 2000; 105: 905-913.

21 Kiefmann R, Islam MN, Lindert J, et al. Paracrine purinergic signaling determines lung endothelial nitric oxide production. Am J Physiol Lung Cell Mol Physiol 2009; 296: L901-L910. proinflammatory signaling by membrane pore formation. Am J Physiol Lung Cell Mol Physiol 2012; 303: L107-L116.

23 Kuebler WM, Ying X, Singh B, et al. Pressure is proinflammatory in lung venular capillaries. J Clin Invest 1999; 104: 495-502.

24 Huertas A, Das SR, Emin M, et al. Erythrocytes induce proinflammatory endothelial activation in hypoxia. Am J Respir Cell Mol Biol 2013; 48: 78-86.

25 Emin MT, Sun L, Huertas A, et al. Platelets induce endothelial tissue factor expression in a mouse model of acid-induced lung injury. Am J Physiol Lung Cell Mol Physiol 2012; 302: L1209-L1220.

chimura H, Parthasarathi K, Quadri S, et al. Mechano-oxidative coupling proinflammatory responses in lung venular capillaries. J Clin Invest 2003; 111: 691-699. Ying X, Minamiya Y, Fu C, et al. $\mathrm{Ca}^{2+}$ waves in lung capillary endothelium. Circ Res 1996 determines the severity of inflammation in mouse lung microvessels. J Clin Invest 2011; 121: 1986-1999.

Islam MN, Das SR, Emin MT, et al. Mitochondrial transfer from bone-marrow-derived stromal cells to pulmonary alveoli protects against acute lung injury. Nat Med 2012; 18: 759-765.

30 Swenson ER, Bärtsch P. High-altitude pulmonary edema. Compr Physiol 2012; 2: 2753-2773.

31 Scherrer U, Vollenweider L, Delabays A, et al. Inhaled nitric oxide for high-altitude pulmonary edema. $N$ Engl Med 1996; 334: 624-629.

32 Grunig E, Weissmann S, Ehlken N, et al. Stress Doppler echocardiography in relatives of patients with idiopathic and familial pulmonary arterial hypertension: results of a multicenter European analysis of pulmonary artery pressure response to exercise and hypoxia. Circulation 2009; 119: 1747-1757.

33 Dehnert C, Mereles D, Greiner S, et al. Exaggerated hypoxic pulmonary vasoconstriction without susceptibility to high altitude pulmonary edema. High Alt Med Biol 2015; 16: 11-17.

34 Bärtsch P, Maggiorini M, Mairbäurl H, et al. Pulmonary extravascular fluid accumulation in climbers. Lancet 2002; 360: 571; author reply 571-572.

35 Bärtsch P, Swenson ER. Clinical practice: acute high-altitude illnesses. N Engl J Med 2013; 368: $2294-2302$.

36 Nana-Sinkam SP, Lee JD, Sotto-Santiago S, et al. Prostacyclin prevents pulmonary endothelial cell apoptosis induced by cigarette smoke. Am J Respir Crit Care Med 2007; 175: 676-685.

37 Bärtsch P, Maggiorini M, Ritter M, et al. Prevention of high-altitude pulmonary edema by nifedipine. $N$ Engl $J$ Med 1991; 325: 1284-1289.

38 Hultgren HN, Grover RF, Hartley LH. Abnormal circulatory responses to high altitude in subjects with a previous history of high-altitude pulmonary edema. Circulation 1971; 44: 759-770.

39 Grunig E, Mereles D, Hildebrandt W, et al. Stress Doppler echocardiography for identification of susceptibility to high altitude pulmonary edema. J Am Coll Cardiol 2000; 35: 980-987.

40 Maggiorini M, Brunner-La Rocca HP, Peth S, et al. Both tadalafil and dexamethasone may reduce the incidence of high-altitude pulmonary edema: a randomized trial. Ann Intern Med 2006; 145: 497-506.

41 Oelz O, Maggiorini M, Ritter M, et al. Nifedipine for high altitude pulmonary oedema. Lancet 1989; 2: 1241-1244.

42 Maggiorini M, Melot C, Pierre S, et al. High-altitude pulmonary edema is initially caused by an increase in capillary pressure. Circulation 2001; 103: 2078-2083.

43 Vock P, Brutsche MH, Nanzer A, et al. Variable radiomorphologic data of high altitude pulmonary edema. Features from 60 patients. Chest 1991; 100: 1306-1311.

44 Hopkins SR, Garg J, Bolar DS, et al. Pulmonary blood flow heterogeneity during hypoxia and high-altitude pulmonary edema. Am J Respir Crit Care Med 2005; 171: 83-87.

45 Dehnert C, Risse F, Ley S, et al. Magnetic resonance imaging of uneven pulmonary perfusion in hypoxia in humans. Am J Respir Crit Care Med 2006; 174: 1132-1138.

46 Swenson ER, Maggiorini M, Mongovin S, et al. Pathogenesis of high-altitude pulmonary edema: inflammation is not an etiologic factor. JAMA 2002; 287: 2228-2235.

47 Schoene RB, Swenson ER, Pizzo CJ, et al. The lung at high altitude: bronchoalveolar lavage in acute mountain sickness and pulmonary edema. J Appl Physiol 1988; 64: 2605-2613.

48 Kubo K, Hanaoka M, Hayano T, et al. Inflammatory cytokines in BAL fluid and pulmonary hemodynamics in high-altitude pulmonary edema. Respir Physiol 1998; 111: 301-310. 
Bärtsch P, Mairbäurl H, Maggiorini M, et al. Physiological aspects of high-altitude pulmonary edema. J Appl Physiol 2005; 98: 1101-1110.

50 Soria R, Egger M, Scherrer U, et al. Pulmonary artery pressure and arterial oxygen saturation in people living at high or low altitude: systematic review and meta-analysis. J Appl Physiol 2016; 121: 1151-1159.

51 Wilkins MR, Aldashev AA, Wharton J, et al. $\alpha 1-A 680 \mathrm{~T}$ variant in GUCY1A3 as a candidate conferring protection from pulmonary hypertension among Kyrgyz highlanders. Circ Cardiovasc Genet 2014; 7: 920-929.

52 Sylvester JT, Shimoda LA, Aaronson PI, et al. Hypoxic pulmonary vasoconstriction. Physiol Rev 2012; 92: 367-520.

53 Wilkins MR, Ghofrani HA, Weissmann N, et al. Pathophysiology and treatment of high-altitude pulmonary vascular disease. Circulation 2015; 131: 582-590.

54 Busch T, Bärtsch P, Pappert D, et al. Hypoxia decreases exhaled nitric oxide in mountaineers susceptible to high-altitude pulmonary edema. Am J Respir Crit Care Med 2001; 163: 368-373.

55 Duplain H, Sartori C, Lepori M, et al. Exhaled nitric oxide in high-altitude pulmonary edema: role in the regulation of pulmonary vascular tone and evidence for a role against inflammation. Am J Respir Crit Care Med 2000; 162: 221-224.

Bailey DM, Dehnert C, Luks AM, et al. High-altitude pulmonary hypertension is associated with a free radical-mediated reduction in pulmonary nitric oxide bioavailability. J Physiol 2010; 588: 4837-4847.

57 Sartori C, Vollenweider L, Loffler BM, et al. Exaggerated endothelin release in high-altitude pulmonary edema. Circulation 1999; 99: 2665-2668

58 Hohenhaus E, Paul A, McCullough RE, et al. Ventilatory and pulmonary vascular response to hypoxia and susceptibility to high altitude pulmonary oedema. Eur Respir J 1995; 8: 1825-1833.

59 Viswanathan R, Jain SK, Subramanian S, et al. Pulmonary edema of high altitude. II. Clinical, aerohemodynamic, and biochemical studies in a group with history of pulmonary edema of high altitude. Am Rev Respir Dis 1969; 100: 334-341.

60 Eldridge MW, Podolsky A, Richardson RS, et al. Pulmonary hemodynamic response to exercise in subjects with prior high-altitude pulmonary edema. J Appl Physiol 1996; 81: 911-921.

61 Dinh-Xuan AT. Endothelial modulation of pulmonary vascular tone. Eur Respir J 1992; 5: 757-762.

62 Vanhoutte PM, Zhao Y, Xu A, et al. Thirty years of saying NO: sources, fate, actions, and misfortunes of the endothelium-derived vasodilator mediator. Circ Res 2016; 119: 375-396.

63 Duong-Quy S, Bei Y, Liu Z, et al. Role of Rho-kinase and its inhibitors in pulmonary hypertension. Pharmaco Ther 2013; 137: 352-364

64 Duong-Quy S, Dao P, Hua-Huy T, et al. Increased Rho-kinase expression and activity and pulmonary endothelial dysfunction in smokers with normal lung function. Eur Respir I 2011; 37: 349-355.

65 Bei Y, Duong-Quy S, Hua-Huy T, et al. Activation of RhoA/Rho-kinase pathway accounts for pulmonary endothelial dysfunction in patients with chronic obstructive pulmonary disease. Physiol Rep 2013; 1: e00105.

66 Weissmann N, Winterhalder S, Nollen M, et al. NO and reactive oxygen species are involved in biphasic hypoxic vasoconstriction of isolated rabbit lungs. Am J Physiol Lung Cell Mol Physiol 2001; 280: L638-L645.

67 Petousi N, Robbins PA. Human adaptation to the hypoxia of high altitude: the Tibetan paradigm from the pregenomic to the postgenomic era. J Appl Physiol 2014; 116: 875-884.

68 Zhao L, Oliver E, Maratou K, et al. The zinc transporter ZIP12 regulates the pulmonary vascular response to chronic hypoxia. Nature 2015; 524: 356-360

69 Stenmark KR, Fagan KA, Frid MG. Hypoxia-induced pulmonary vascular remodeling: cellular and molecular mechanisms. Circ Res 2006; 99: 675-691.

70 Stenmark KR, McMurtry IF. Vascular remodeling versus vasoconstriction in chronic hypoxic pulmonary hypertension: a time for reappraisal? Circ Res 2005; 97: 95-98.

71 Abe K, Shinoda M, Tanaka M, et al. Haemodynamic unloading reverses occlusive vascular lesions in severe pulmonary hypertension. Cardiovasc Res 2016; 111: 16-25.

72 McLaughlin VV, Shah SJ, Souza R, et al. Management of pulmonary arterial hypertension. J Am Coll Cardiol 2015; 65: 1976-1997.

73 Humbert M, Sitbon O, Chaouat A, et al. Survival in patients with idiopathic, familial, and anorexigen-associated pulmonary arterial hypertension in the modern management era. Circulation 2010; 122: 156-163.

74 Simonneau G, Gatzoulis MA, Adatia I, et al. Updated clinical classification of pulmonary hypertension. J Am Coll Cardiol 2013; 62: Suppl. 25, D34-D41.

75 Guignabert C, Tu L, Girerd B, et al. New molecular targets of pulmonary vascular remodeling in pulmonary arterial hypertension: importance of endothelial communication. Chest 2015; 147: 529-537.

76 Huertas A, Perros F, Tu L, et al. Immune dysregulation and endothelial dysfunction in pulmonary arterial hypertension: a complex interplay. Circulation 2014; 129: 1332-1340.

77 Le Hiress M, Tu L, Ricard N, et al. Proinflammatory signature of the dysfunctional endothelium in pulmonary hypertension. Role of the macrophage migration inhibitory factor/CD74 complex. Am J Respir Crit Care Med 2015; 192: 983-997.

78 Tu L, Dewachter L, Gore B, et al. Autocrine fibroblast growth factor-2 signaling contributes to altered endothelial phenotype in pulmonary hypertension. Am J Respir Cell Mol Biol 2011; 45: 311-322.

79 Tu L, De Man FS, Girerd B, et al. A critical role for p130Cas in the progression of pulmonary hypertension in humans and rodents. Am J Respir Crit Care Med 2012; 186: 666-676.

80 de Man FS, Tu L, Handoko ML, et al. Dysregulated renin-angiotensin-aldosterone system contributes to pulmonary arterial hypertension. Am J Respir Crit Care Med 2012; 186: 780-789.

81 Huertas A, Tu L, Gambaryan N, et al. Leptin and regulatory T-lymphocytes in idiopathic pulmonary arterial hypertension. Eur Respir J 2012; 40: 895-904.

82 Huertas A, Tu L, Thuillet R, et al. Leptin signalling system as a target for pulmonary arterial hypertension therapy. Eur Respir J 2015; 45: 1066-1080.

83 Huertas A, Phan C, Bordenave J, et al. Regulatory T cell dysfunction in idiopathic, heritable and connective tissue-associated pulmonary arterial hypertension. Chest 2016; 149: 1482-1493. 
Ricard N, Tu L, Le Hiress M, et al. Increased pericyte coverage mediated by endothelial-derived fibroblast growth factor-2 and interleukin-6 is a source of smooth muscle-like cells in pulmonary hypertension. Circulation 2014; 129: $1586-1597$.

Montani D, Chaumais MC, Guignabert C, et al. Targeted therapies in pulmonary arterial hypertension. Pharmacol Ther 2014; 141: 172-191.

Blanco I, Piccari L, Barbera JA. Pulmonary vasculature in COPD: the silent component. Respirology 2016; 21: 984-994.

Hilde JM, Skjorten I, Hansteen V, et al. Haemodynamic responses to exercise in patients with COPD. Eur Respir J 2013; 41: 1031-1041.

Portillo K, Torralba Y, Blanco I, et al. Pulmonary hemodynamic profile in chronic obstructive pulmonary disease. Int J Chron Obstruct Pulmon Dis 2015; 10: 1313-1320.

Santos S, Peinado VI, Ramirez J, et al. Characterization of pulmonary vascular remodelling in smokers and patients with mild COPD. Eur Respir J 2002; 19: 632-638.

Barbera JA, Peinado VI, Santos S, et al. Reduced expression of endothelial nitric oxide synthase in pulmonary arteries of smokers. Am J Respir Crit Care Med 2001; 164: 709-713.

Adir Y, Shachner R, Amir O, et al. Severe pulmonary hypertension associated with emphysema: a new phenotype? Chest 2012; 142: 1654-1658.

Peinado VI, Gomez FP, Barbera JA, et al. Pulmonary vascular abnormalities in chronic obstructive pulmonary disease undergoing lung transplant. J Heart Lung Transplant 2013; 32: 1262-1269.

Chaouat A, Bugnet AS, Kadaoui N, et al. Severe pulmonary hypertension and chronic obstructive pulmonary disease. Am J Respir Crit Care Med 2005; 172: 189-194.

Dominguez-Fandos D, Valdes C, Ferrer E, et al. Sildenafil in a cigarette smoke-induced model of COPD in the guinea-pig. Eur Respir J 2015; 46: 346-354.

Seimetz M, Parajuli N, Pichl A, et al. Cigarette smoke-induced emphysema and pulmonary hypertension can be prevented by phosphodiesterase 4 and 5 inhibition in mice. PloS One 2015; 10: e0129327.

Ahluwalia A, Foster P, Scotland RS, et al. Anti-inflammatory activity of soluble guanylate cyclase: cGMP-dependent down-regulation of P-selectin expression and leukocyte recruitment. Proc Natl Acad Sci USA 2004; 101: 1386-1391.

Ataga KI, Moore CG, Jones S, et al. Pulmonary hypertension in patients with sickle cell disease: a longitudinal study. Br J Haematol 2006; 134: 109-115.

De Castro LM, Jonassaint JC, Graham FL, et al. Pulmonary hypertension associated with sickle cell disease: clinical and laboratory endpoints and disease outcomes. Am J Hematol 2008; 83: 19-25.

Gladwin MT, Sachdev V, Jison ML, et al. Pulmonary hypertension as a risk factor for death in patients with sickle cell disease. N Engl J Med 2004; 350: 886-895.

Machado RF, Anthi A, Steinberg MH, et al. N-terminal pro-brain natriuretic peptide levels and risk of death in sickle cell disease. JAMA 2006; 296: 310-318.

Parent F, Bachir D, Inamo J, et al. A hemodynamic study of pulmonary hypertension in sickle cell disease. N Engl J Med 2011; 365: 44-53.

Gladwin MT. Cardiovascular complications and risk of death in sickle-cell disease. Lancet 2016; 387: 2565-2574.

Reiter CD, Wang X, Tanus-Santos JE, et al. Cell-free hemoglobin limits nitric oxide bioavailability in sickle-cell disease. Nat Med 2002; 8: 1383-1389.

Rother RP, Bell L, Hillmen P, et al. The clinical sequelae of intravascular hemolysis and extracellular plasma hemoglobin: a novel mechanism of human disease. JAMA 2005; 293: 1653-1662.

Gladwin MT, Ofori-Acquah SF. Erythroid DAMPs drive inflammation in SCD. Blood 2014; 123: 3689-3690.

Donadee C, Raat NJ, Kanias T, et al. Nitric oxide scavenging by red blood cell microparticles and cell-free hemoglobin as a mechanism for the red cell storage lesion. Circulation 2011; 124: 465-476.

Peinado VI, Ramirez J, Roca J, et al. Identification of vascular progenitor cells in pulmonary arteries of patients with chronic obstructive pulmonary disease. Am J Respir Cell Mol Biol 2006; 34: 257-263.

Gordon C, Gudi K, Krause A, et al. Circulating endothelial microparticles as a measure of early lung destruction in cigarette smokers. Am J Respir Crit Care Med 2011; 184: 224-232.

Thomashow MA, Shimbo D, Parikh MA, et al. Endothelial microparticles in mild chronic obstructive pulmonary disease and emphysema. The multi-ethnic study of atherosclerosis chronic obstructive pulmonary disease study. Am J Respir Crit Care Med 2013; 188: 60-68.

Takahashi T, Kubo $\mathrm{H}$. The role of microparticles in chronic obstructive pulmonary disease. Int J Chron Obstruct Pulmon Dis 2014; 9: 303-314.

Feng B, Chen Y, Luo Y, et al. Circulating level of microparticles and their correlation with arterial elasticity and endothelium-dependent dilation in patients with type 2 diabetes mellitus. Atherosclerosis 2010; 208: 264-269.

Palange P, Testa U, Huertas A, et al. Circulating haemopoietic and endothelial progenitor cells are decreased in COPD. Eur Respir J 2006; 27: 529-541.

Pizarro S, Garcia-Lucio J, Peinado VI, et al. Circulating progenitor cells and vascular dysfunction in chronic obstructive pulmonary disease. PloS One 2014; 9: e106163.

Fadini GP, Schiavon M, Cantini M, et al. Circulating progenitor cells are reduced in patients with severe lung disease. Stem Cells 2006; 24: 1806-1813.

Huertas A, Testa U, Riccioni R, et al. Bone marrow-derived progenitors are greatly reduced in patients with severe COPD and low-BMI. Respir Physiol Neurobiol 2010; 170: 23-31.

Hill JM, Zalos G, Halcox JP, et al. Circul
risk. N Engl J Med 2003; 348: 593-600

Basile DP, Yoder MC. Renal endothelial dysfunction in acute kidney ischemia reperfusion injury. Cardiovasc Hematol Disord Drug Targets 2014; 14: 3-14.

Pelosi E, Castelli G, Testa U. Endothelial progenitors. Blood Cells Mol Dis 2014; 52: 186-194.

Ingram DA, Mead LE, Tanaka $\mathrm{H}$, et al. Identification of a novel hierarchy of endothelial progenitor cells using human peripheral and umbilical cord blood. Blood 2004; 104: 2752-2760.

Yoder MC, Mead LE, Prater D, et al. Redefining endothelial progenitor cells via clonal analysis and hematopoietic stem/progenitor cell principals. Blood 2007; 109: 1801-1809. 